\section{COPD: Kurz wirksame Beta-Agonisten oft übermäßig gebraucht}

Fan VS et al. Overuse of short-acting beta-agonist brochodilators in COPD during periods of clinical stability. Respir Med 2016; 116: 100 106

Ein übermäßiger Gebrauch kurz wirksamer Beta-Agonisten kann zu einer höheren Rate an unerwünschten Wirkungen führen. Beim Asthma ist dieses Phänomen beschrieben, doch bei der COPD ist hierzu nur wenig bekannt. V. S. Fan et al. gingen dieser Frage nun nach.

Die Autoren nutzten für ihre Untersuchung Daten einer prospektiven Beobachtungsstudie ambulanter Patienten mit COPD, die sich über 3 Monate erstreckte. Teilnahmebedingungen waren neben einer COPD eine FEV1 von maximal $80 \%$, der Gebrauch eines inhalativen kurz wirksamen Beta-Agonisten (SABA), ein Nikotinkonsum von mehr als 10 Packyears ohne aktuellen Konsum sowie ein Alter über 49,5 Jahre. In den letzten vier Wochen vor Studienbeginn durfte es zudem zu keiner Exazerbation gekommen sein. Eingangs unterzogen sich die Teilnehmer einer Spirometrie und füllten Fragebögen zu repiratorischen Symptomen aus. Sie erhielten für die Studienperiode einen Schrittzähler sowie einen elektronischen Sensor, der den Verbrauch des SABA über den Inhaler erfasste. Zusätzlich dokumentierten sie ihren Medikamentenkonsum in einem Tagebuch. Ein übermäßiger Gebrauch des SABA war definiert als mehr als 8 Hübe am Tag während einer klinisch stabilen Situation.

In die Analyse gingen 32 Teilnehmer ein, die Albuterol als SABA verwendeten. Von diesen kam es bei 15 zu mindestens einem übermäßigen Gebrauch mit einem Mittel von 8,6 Hüben, bei 6 von ihnen fand sich ein übermäßiger Gebrauch an mindestens der Hälfte der Studientage. Verglichen mit Patienten ohne übermäBigen Gebrauch berichteten diejenigen mit über vermehrte Dyspnoe (2,7 vs. 1,9 auf der modifizierten Medical Research
Council Dyspnea Scale), nutzten zu einer höheren Rate Heim-Sauerstoff (67\% vs. $29 \%$ ) und wurden zu einem größeren Anteil mit einer Maximaltherapie (lang wirksamer Beta-Agonist, lang wirksamer Muskarin-Antagonist und inhalatives Steroid; $40 \%$ vs. $6 \%$ ) behandelt. $27 \%$ der Patienten mit übermäßigem Gebrauch hatten jedoch keine leitliniengerechte Therapie der COPD.

FAZIT

Ein übermäßiger Gebrauch von SABA war bei Patienten mit COPD häufig und mit einer schwereren Erkrankung sowie vermehrten Symptomen assoziiert, so die Autoren.

Dr. med. Johannes Weiß, Bad Kissingen 